\title{
Ultrafast dynamics in QD based photoelectrochemical cells
}

Honarfar, Alireza; Mourad, Hassan; Abdellah, Mohamed; Chabera, Pavel; Pankratova, Galina; Gorton, Lo; Zheng, Kaibo; Pullerits, Tönu

Published in:

Physical Chemistry of Semiconductor Materials and Interfaces XVIII

Link to article, DOI:

$10.1117 / 12.2529771$

Publication date:

2019

Document Version

Publisher's PDF, also known as Version of record

Link back to DTU Orbit

Citation (APA):

Honarfar, A., Mourad, H., Abdellah, M., Chabera, P., Pankratova, G., Gorton, L., Zheng, K., \& Pullerits, T. (2019). Ultrafast dynamics in QD based photoelectrochemical cells. In C. N., D. C., H. A. B., \& F. D. (Eds.) Physical Chemistry of Semiconductor Materials and Interfaces XVIII (Vol. 11084). SPIE - International Society for Optical Engineering. Proceedings of SPIE - The International Society for Optical Engineering https://doi.org/10.1117/12.2529771

\section{General rights}

Copyright and moral rights for the publications made accessible in the public portal are retained by the authors and/or other copyright owners and it is a condition of accessing publications that users recognise and abide by the legal requirements associated with these rights.

- Users may download and print one copy of any publication from the public portal for the purpose of private study or research.

- You may not further distribute the material or use it for any profit-making activity or commercial gain

- You may freely distribute the URL identifying the publication in the public portal 


\title{
Ultrafast dynamics in QD based photoelectrochemical cells
}

\begin{abstract}
Alireza Honarfar, Hassan Mourad, Mohamed Abdellah, Pavel Chabera, Galina Pankratova, et al.
\end{abstract}

Alireza Honarfar, Hassan Mourad, Mohamed Abdellah, Pavel Chabera, Galina Pankratova, Lo Gorton, Kaibo Zheng, Tönu Pullerits, "Ultrafast dynamics in QD based photoelectrochemical cells," Proc. SPIE 11084, Physical Chemistry of Semiconductor Materials and Interfaces XVIII, 110840L (9 September 2019); doi: 10.1117/12.2529771

Event: SPIE Nanoscience + Engineering, 2019, San Diego, California, United States 


\title{
Ultrafast dynamics in QD based photoelectrochemical cells
}

\author{
Alireza Honarfar ${ }^{\mathrm{a}}$, Hassan Mourad ${ }^{\mathrm{a}}$, Mohamed Abdellah ${ }^{\mathrm{a}, \mathrm{d}}$, Pavel Chabera ${ }^{\mathrm{a}}$, Galina Pankratova ${ }^{\mathrm{b}}$, \\ Lo Gorton $^{\mathrm{b}}$, Kaibo Zheng ${ }^{\mathrm{a}, \mathrm{c}}$, Tõnu Pullerits*a \\ a'Department of Chemical Physics and NanoLund, Lund University, Box 124, 22100 Lund, Sweden; \\ ${ }^{\mathrm{b}}$ Biochemistry and Structural Biology, Lund University, Box 124, 2200 Lund, Sweden; ${ }^{\mathrm{C}}$ Department \\ of Chemistry, Technical University of Denmark, DK-2800 Kongens Lyngby, Denmark \\ dÅngstrom Laboratory, Department of Chemistry, Uppsala University, Box 523, 75120 Uppsala, \\ Sweden
}

\begin{abstract}
We have prepared electrodes for photo-electro-chemical cells which have enabled to pre-charge colloidal quantum dots in well-controlled fashion. Femtosecond transient absorption measurements were carried out revealing clear speed-up of the photo-induced charge carrier dynamics, particularly the recombination. Such studies allow to understand the behavior of light harvesting materials in operational conditions of optoelectronic devices giving new recipes for improvements.
\end{abstract}

Keywords: Femtosecond spectro-electrochemistry, quantum dots, CdSe, trion, exciton

\section{INTRODUCTION}

Colloidal semiconductor nanocrystals, so called quantum dots (QDs) and their assemblies have been extensively studied for light harvesting and photo-catalytic applications due to their high absorption coefficient, size-tunable optical band gap and possible multiple exciton generation. ${ }^{1-3}$ Recent years have shown the potential of QDs from the new perovskite solar energy material for optoelectronics applications. ${ }^{4-8}$ Such applications rely on separation of photo-generated electron-hole pairs for efficient photon to electron conversion, which is typically carried out through charge transfer from the QDs to electronically coupled electron (or hole) acceptors. Charge transfer in QD-acceptor systems have been extensively investigated by time-resolved spectroscopies.9-13 However, most of the earlier studies have been implemented in half-cell systems (i.e. only photoanodes or photocathodes) in open-circuit condition. Under such circumstances, the initial conditions of the measured QDs is zero charge despite of the photoinduced charge separation at the QD/acceptor interface during the measurements. In full devices, however, injection or extraction of charges by an external electric field to/from the electrodes is essential for device operation. Consequently, the QD-acceptor system is usually charged during the functioning of the device. Previous studies by optical spectroscopy, ${ }^{14}$ electrochemistry ${ }^{15-18}$ and combination of both ${ }^{18-21}$ show that the presence of extra charges does influence the electronic structure of the QDs. For instance, one electron injection into the conduction band (CB) of the nanoparticles provides a chemical reduction significantly affecting the steady state absorption at the excitonic band ${ }^{22}$ and the addition of two electrons to the CB simultaneously by using electrochemistry makes the absorption of the excitonic band completely bleached. In semiconductors the change of optical properties due to the Fermi level shift toward the carrier bands by using high doping concentrations is explained as the Burstein-Moss effect, which recently has been used for evaluation of the absolute position of band edges of semiconductor nanoparticles ${ }^{23}$ However, recent studies by time resolved spectroscopy revealed very unconventional excited state photophysics of charged QDs. ${ }^{24}$ It was explained via so called trions, which induce fast non radiative recombination of the charged exciton, electron-hole pair together with an additional electron or hole, similar to the Auger process. By considering these effects, one can expect very different charge transfer dynamics in QD-acceptor systems when QDs are pre-charged due to an external applied electric field compared with open-circuit conditions. Previously it was reported that, filling trap states by controlled the potential in a QDs assembly results in enhanced charge transfer between nanoparticles. ${ }^{25}$ In this work we will investigate how the charge transfer dynamics in the QD-acceptors system is influenced in case the nanoparticles are fully charged by application of an external bias. We will probe the dynamics by using femtosecond electrochemical time resolved transient absorption spectroscopy (FECTA). We demonstrated clear changes in ultrafast exciton dynamics in QDs attached to TiO2 under the applied potential. The findings give new hints for rational design of QD-based optoelectronic devices.

*tonu.pullerits@chemphys.lu.se; phone +4646 2228131; www.chemphys.lu.se

Physical Chemistry of Semiconductor Materials and Interfaces XVIII, edited by Christian Nielsen, Daniel Congreve, Hugo A. Bronstein, Felix Deschler, Proc. of SPIE Vol. 11084, 110840L (C) 2019 SPIE · CCC code: 0277-786X/19/\$21 - doi: 10.1117/12.2529771 


\section{MATERIALS AND METHODS}

Octadecene (ODE), Cadmium oxide (CdO), Selenium powder (Se), Oleic acid (OLEA), Trioctylphosphine (TOP), Dichloromethane (DCM), Methanol (MEOH), Acetone, 3-mercaptopropionic acid (3-MPA), Fluorine doped tin oxide on glass (FTO), tetrabutylammonium hexafluorophosphate (TBAPF6) were purchased from Sigma-Aldrich and were used as received without any purification or distillation. $3 \AA$ molecular sieves powder were activated by heating and vacuum then used for drying and storage of solvents if needed.

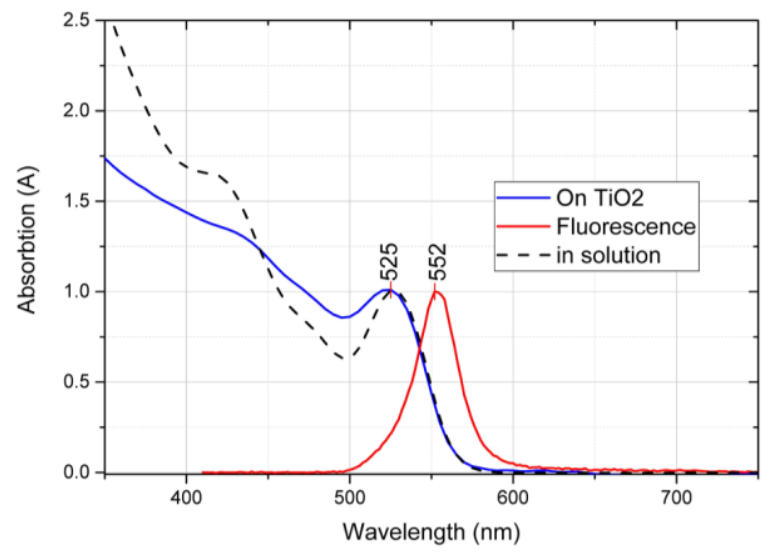

Figure 1. Absorption and photoexcited luminescence spectra of the CdSe QDs used in this study. Also the absorption spectrum of the QD- $\mathrm{TiO}_{2}$ film is shown.

Conventional hot injection method was used for QD synthesis. $1500 \mathrm{mgr}$ of CdO was dissolved in $7 \mathrm{ml}$ of OLEA and 50 $\mathrm{ml}$ of ODE at $270^{\circ} \mathrm{C}$ in a three-neck flask. Selenium precursor was prepared by sonication of Se powder in $10 \mathrm{ml}$ of ODE for $10 \mathrm{~min}$ in an Ar purged flask then by adding $0.5 \mathrm{~mL}$ of trioctylphosphine and then stirred until it became a transparent solution. When the Cd precursor solution became clearly transparent the temperature was lowered to $240^{\circ} \mathrm{C}$, then the Se solution was quickly injected at $240^{\circ} \mathrm{c}$ and stirred for $2 \mathrm{~min}$. Then the flask was removed from the heater and the hot solution was quickly poured into a metallic bucket, which was placed in a cold bath filled with dry ice. This immediate cooling proved to be very efficient to keep the size distribution of the nanoparticles very narrow. The synthesized CdSe QDs were characterized to ensure the quality, size distribution and shape of the nanoparticles. The absorption spectrum shows a very distinctive excitonic peak around $545 \mathrm{~nm}$, see Figure 1. From the excitonic peak, we evaluated the size of the QDs to be $3.5 \mathrm{~nm}$. Photoluminescence of the QDs excited at $400 \mathrm{~nm}$, shows a narrow emission band at $550 \mathrm{~nm}$ with FWHM $29 \mathrm{~nm}$. The capping ligand was changed to 3-MPA. The FTO slides were coated with TiO2 paste, sintered at $480^{\circ} \mathrm{C}$ and cooled overnight. Films, see Figure 2, were prepared by soaking TiO2 coated FTO slides in a MPA capped QDs solution at basic $\mathrm{pH}$ for more than $24 \mathrm{~h}$, then washed with distilled water and heated to $150^{\circ} \mathrm{C}$. Films were stored in a vacuum desiccator over drying agents. Films were kept in dark, since photo-degradation could be observed for samples under illumination due to charge transfer. ${ }^{26}$

3 electrode system was used for electrochemistry. $\mathrm{TiO}_{2}$ coated FTO glass was used as working electrode, Pt wire as counter electrode and leak-less $\mathrm{Ag} / \mathrm{AgCl}$ as reference electrode. The electrochemical cell was made from glass with a 3 $\mathrm{mm}$ spacer, see Figure 2. For electrolyte solution 0.1M tetrabutylammonium hexafluorophosphate in DCM was used. Electrolyte selection is a critical decision, because adsorption and intercalation of electrolyte molecules into the nanoparticles shows significant effects on the electrochemistry. ${ }^{27}$

Output of amplified laser system provided $796 \mathrm{~nm}, 6 \mathrm{~mJ}, 100 \mathrm{fs}$ pulses which used to generate both pump and probe light. Second harmonic of the fundamental laser was used as the pump pulses with central wavelength $400 \mathrm{~nm}$. White light generated in $\mathrm{CaF} 2$ crystal was used as probe $(350 \mathrm{~nm}$ to $1200 \mathrm{~nm})$. The collected data is a 2D map consisting of the 
evolution of the absorption difference in time after excitation by pump pulse. In our measurement the negative signal corresponds to the ground state bleach.

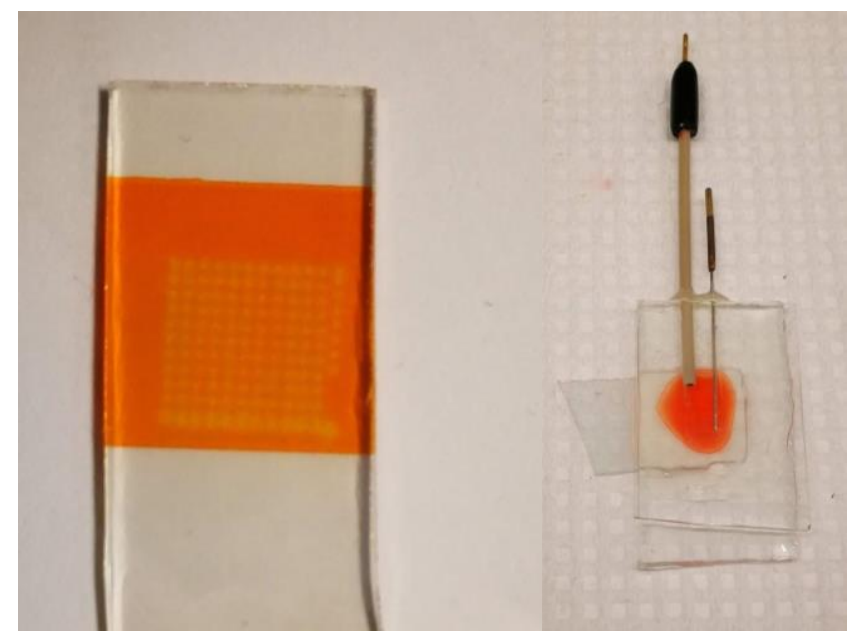

Figure 2. Left: film of CdSe QDs on TiO2 coated FTO. The dotted matrix in the film represents the photodegradation of QDs due to charge transfer during TA measurements. Right: homemade electrochemical cell consisting of $\mathrm{TiO}_{2}$ coated FTO as working electrode, leak-less $\mathrm{Ag} / \mathrm{AgCl}$ reference electrode and a $\mathrm{Pt}$ wire as counter electrode.

\section{RESULTS AND DISCUSSION}

The CdSe QDs films on $\mathrm{TiO}_{2}$, can be considered as two semiconductors, which are attached by 3MPA as linker. In equilibrium the Fermi levels of the semiconductors that are in contact with each other, will be equal through the junction. In that respect we can consider the $\mathrm{FTO}-\mathrm{TiO}_{2}$ layer all together to be as one system with a common Fermi level. If a bias voltage is applied, it causes a shift in the electrodes' Fermi level relative to each other, which causes band bending at the junction of two semiconductors and consequently forces charges to move through the junction to reach a new equilibrium, which can be measured as an electrical current. Since in electrochemistry the potential is measured versus a reference electrode, which has a fixed potential over a wide range, negative potentials can be considered as an increase in the $\mathrm{TiO}_{2}$-FTO Fermi level, such that electrons in $\mathrm{TiO}_{2}-\mathrm{FTO}$ can reach energy levels equal or higher than those of $\mathrm{CdSe}$ $\mathrm{CB}$. So, if the negative potential is high enough, electrons can leave $\mathrm{FTO}^{-\mathrm{TiO}_{2}}$ and enter the CdSe QDs. At positive potentials by lowering the Fermi level until carriers in $\mathrm{FTO}^{-\mathrm{TiO}_{2}}$ have lower energy than their counterparts in the $\mathrm{CdSe}$ valence band (VB), electrons can leave CdSe VB and migrate into TiO2-FTO. When the Fermi level is moved so high (down) that it merges with the conduction (valence) band then the semiconductor can act like a $\mathrm{N}$ type (P type) semiconductor with very high doping.

Femtosecond transient absorption spectroscopy (TA) was used to probe the excited state dynamics of the QDs under the applied potentials. Global analysis and Single Value Decomposition (SVD) fitting of the TA data were used to quantify the population and depopulation pathways of the excited states.

As a reference, first the transient absorption measurements of the CdSe QDs in solution and on $\mathrm{TiO}_{2}$ film were carried out, see Figure 3. The TA spectra consist of a negative bleach band with a maximum at $550 \mathrm{~nm}$ corresponding to the state filling of band edge after excitation and thermalization of the charges. There also exists a weak broad positive signal from $650 \mathrm{~nm}$ to $1100 \mathrm{~nm}$ attributed to the excited state absorption (ESA). From the spectrograms it is already clear that the bleach signal of the QDs attached to the $\mathrm{TiO}_{2}$ acceptor is decaying significantly faster. This behavior is expected owing to the charge carrier injection from the QD donor to the acceptor. The global analyses of the decay provide 5 components. The shortest subpicosecond component is clearly the hot carrier relaxation. The two picosecond components of low amplitude most likely correspond to a minor proportion of QDs with traps while the ns components correspond to the lifetime of the excited state. After attachment of the QDs the hot carrier relaxation remains unchanged while the ps components' amplitude is significantly increased. The three components of about 2, 50 and $600 \mathrm{ps}$ have rather similar spectrum and amplitude. The two longer ones we assign to the electron injection while the 2 ps component 
can be either injection or partially due to the surface defects generated by the attachments of the QDs. The ns component can be the minor proportion of unattached QDs or reflects the back electron transfer to the QDs.

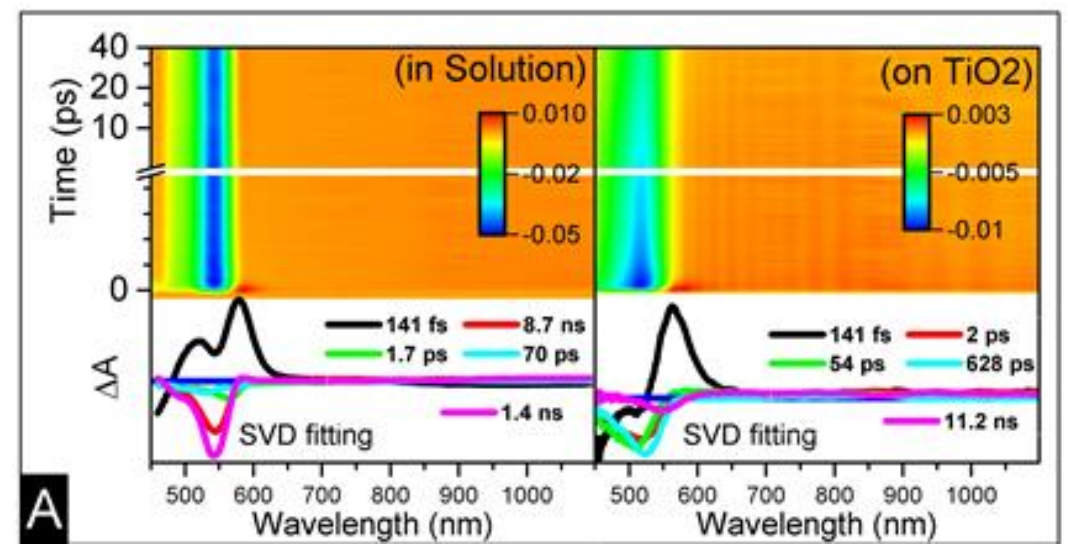

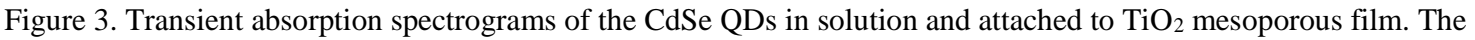
amplitude spectra of the kinetic components obtained by global analyses using singular value decomposition are also provided.

By application of negative potentials, when the voltage is lower than the band edge potential of the QDs no significant changes were observable. This means that no significant charging of the QDs due to the electron transfer from $\mathrm{TiO}_{2}$ occurs since the electrochemical driving force is insufficient to overcome the potential barrier. When the negative voltage reaches $-1.5 \mathrm{~V}$ and higher, however, we observe significant increase of the band edge ground state bleach amplitude at $500 \mathrm{~nm}$, see Figure 4.

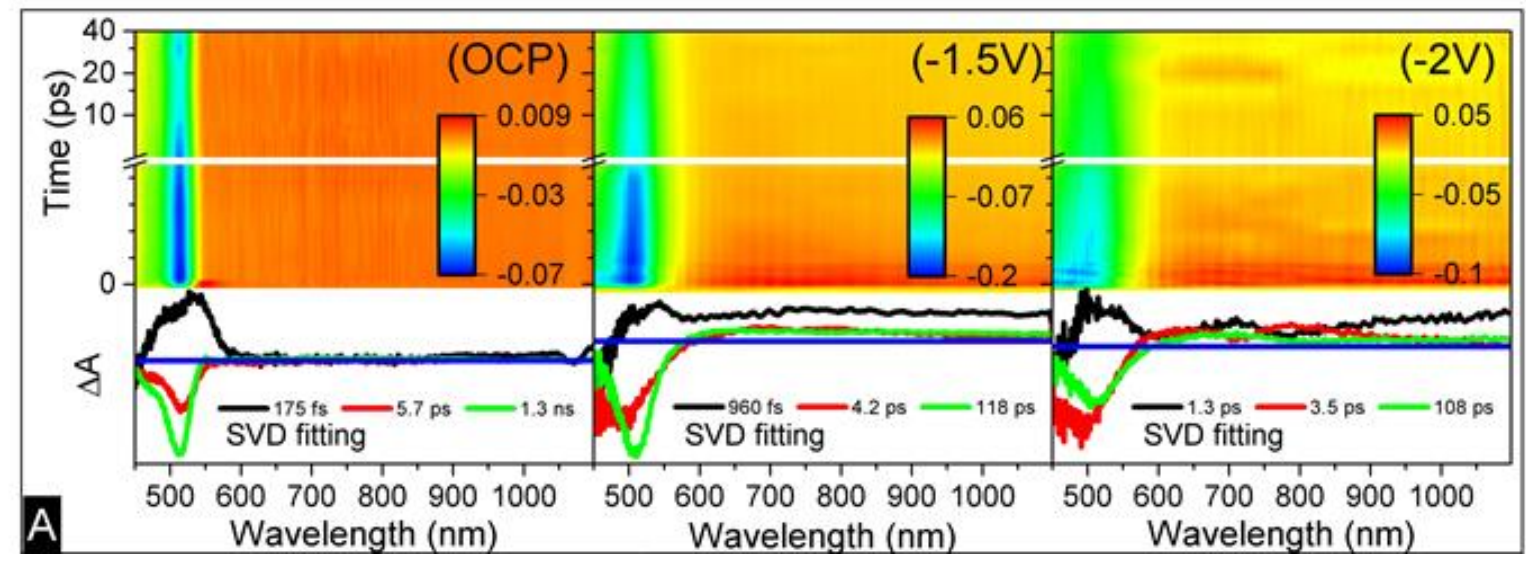

Figure 4. Transient absorption spectrograms of the CdSe QDs attached to $\mathrm{TiO}_{2}$ mesoporous film at different applied electrochemical potential. The amplitude spectra of the kinetic components obtained by global analyses using singular value decomposition are also provided.

We interpret the spectral feature of the $\mathrm{QD}-\mathrm{TiO}_{2}$ at high negative applied voltages by the following model. If the applied negative potential is so high that the Fermi level in the $\mathrm{CB}$ of $\mathrm{TiO}_{2}$ is higher than the $\mathrm{CB}$ edge in CdSe QDs, electrons will move into the QDs. The charges will first fill the doubly degenerate band edge states. If now the pump pulse generates an electron-hole pair, the band-edge transition would be saturated. The corresponding bleach effect would be stronger. We can also see that the bleach decay dynamics becomes significantly faster which we explain by the decay of negative trion. Based on the negative trion mechanism we propose that due to the electrochemical band filling in $\mathrm{CB}$ of the QDs, while photogenerated electrons are decaying, some electrons gain enough energy to jump to higher excited states of the QDs, which gives broadband featureless absorption in the IR region. Such positive absorption is so broad that it cannot be attributed to the Stark shift from the excitation of the QDs. In addition, such a featureless board positive ESA is very similar to the state filling of free charges in the CB of perovskite QDs but with much lower exciton binding 
energy. ${ }^{28}$ Since $\mathrm{TiO}_{2}$ has more density of states these electrons in the higher excited states of the QDs have a higher probability of being trapped in the excited states of $\mathrm{TiO}_{2}$ and follow their relaxation in $\mathrm{TiO}_{2}$, which leads to separation of charges. We point out that the holes cannot transport through the TiO2 barrier at these conditions.

In case of positive bias (data not shown) the behavior is different. We first observed that the ground state bleach is significantly diminished with positive applied voltage. Such suppression on the band edge bleach can be attributed to an ESA signal under the bleach band which suppresses the signal amplitude. The signal corresponds to the transition from the deeper VB levels to the band edge holes. The decay of the bleach band becomes slower with positive voltages, which is due to the evacuated trap states at the QDs interface. The longer wavelength ESA becomes negligible at positive voltage. Unfortunately, the hole dynamics are not clearly visible in the $\mathrm{TA}$ measurement and $\mathrm{TiO}_{2}$ is also considered to be mainly a hole blocking layer, which makes it difficult to conclude the exact hole dynamics from these measurements. The holes in VB have a small energy level splitting and much larger density of states in comparison with the electrons in $\mathrm{CB}$ and the hole transfer is not energetically favorable.

\section{CONCLUSIONS}

We have demonstrated clear electrochemical charging of colloidal QDs and related trion effects in photoinduced dynamics. Such trions would negatively influence most of the optoelectronic devices. In order to avoid them, proper trion neutralization strategies need to be implemented in real life applications of QDs.

\section{REFERENCES}

[1] Zídek, K., Zheng, K., Ponseca, C. S., Messing, M. E., Wallenberg, L. R., Chábera, P., Abdellah, M., Sundström, V., Pullerits, T., "Electron transfer in quantum-dot-sensitized $\mathrm{ZnO}$ nanowires: ultrafast time-resolved absorption and terahertz study. J. Am. Chem. Soc. 134(29), 12110-12117 (2012).

[2] Zheng, K., Karki, K., Žídek, K., Pullerits, T., "Ultrafast photoinduced dynamics in quantum dot-based systems for light harvesting," Nano Res. 8(7), 2125-2142 (2015).

[3] Žídek, K., Zheng, K., Abdellah, M., Lenngren, N., Chábera, P., Pullerits, T., "Ultrafast dynamics of multiple exciton harvesting in the CdSe-ZnO system: electron injection versus Auger recombination," Nano Lett.12(12), 6393-6399 (2012).

[4] Zheng, K., Zhu, Q., Abdellah, M., Messing, M. E., Zhang, W., Generalov, A. V., Niu, Y., Ribaud, L., Canton, S. E., Pullerits, T., "Exciton binding energy and the nature of emissive states in organometal halide perovskites," J. Phys. Chem. Lett. 6(15), 2969-2975 (2015).

[5] Zheng, K., Žídek, K., Abdellah, M., Messing, M. E., Al-Marri, M. J., Pullerits, T., "Trap states and their dynamics in organometal halide perovskite nanoparticles and bulk crystals," J. Phys. Chem. C 120(5), 30773084 (2016).

[6] Chen, J., Žídek, K., Chabera, P., Liu, D., Cheng, P., Nuuttila, L., Al-Marri, M. J., Lehtivuori, H., Messing, M. E., Han, K., Zheng, K., Pullerits, T., "Size and wavelength dependent two-photon absorption cross-section of $\mathrm{CsPbBr}_{3}$ perovskite quantum dots," J. Phys. Chem. Lett. 8(10), 2316-2321 (2017).

[7] Becker, C., Burger, S., Barth, C., Manley, P., Jäger, K., Eisenhauer, D., Köppel, G., Chabera, P., Chen, J., Zheng, K., Pullerits, T., "Nanophotonic-enhanced two-photon-excited photoluminescence of perovskite quantum dots," ACS Photonics 5(11), 4668-4676 (2018).

[8] Chen, J., Messing, M. E., Zheng, K., Pullerits, T., "Cation-dependent hot carrier cooling in halide perovskite nanocrystals," J. Am. Chem. Soc. 141(8), 3532-3540 (2019).

[9] Abdellah, M., Karki, K. J., Lenngren, N., Zheng, K., Pascher, T., Yartsev, A., Pullerits, T., "Ultra long-lived radiative trap states in CdSe quantum dots," J. Phys. Chem. C 118(37), 21682-21686 (2014).

[10]Zheng, K., Źídek, K., Abdellah, M., Zhang, W., Chábera, P., Lenngren, N., Yartsev, A., Pullerits, T., "Ultrafast charge transfer from CdSe quantum dots to p-type NiO: hole injection vs hole trapping," J Phys Chem C. 2014;118: 18462-18471.

[11] Abdellah, M., Žídek, K., Zheng, K., Chábera, P., Messing, M. E., Pullerits, T., "Balancing electron transfer and surface passivation in gradient $\mathrm{CdSe} / \mathrm{ZnS}$ core-shell quantum dots attached to $\mathrm{ZnO}$," J. Phys. Chem. Lett. 4(11), 1760-1765 (2013). 
[12] Hansen, T., Žídek, K., Zheng, K., Abdellah, M., Chábera, P., Persson, P., Pullerits, T., "Orbital topology controlling charge injection in quantum-dot-sensitized solar cells,” J. Phys. Chem. Lett. 5(7), 1157-1162 (2014).

[13] Abdellah, M., Poulsen, F., Zhu, Q., Zhu, N., Zidek, K., Chabera, P., Corti, A., Hansen, T., Chi, Q., Canton, S. E., Zheng, K., Pullerits, T., "Drastic difference between hole and electron injection through the gradient shell of $\mathrm{Cd}_{\mathrm{x}} \mathrm{Se}_{\mathrm{y}} \mathrm{Zn}_{1-\mathrm{x}} \mathrm{S}_{1-\mathrm{y}}$ quantum dots," Nanoscale 9(34), 12503-12508 (2017).

[14] Woggon, U., Wind, O., Gindele, F., Tsitsishvili, E., Müller, M., "Optical transitions in CdSe quantum dots: From discrete levels to broad gain spectra," J. Lumin. 70(1), 269-280 (1996).

[15] Guyot-Sionnest, P., "Charging colloidal quantum dots by electrochemistry," Microchim. Acta.160(3), 309-314 (2008).

[16] Haram, S. K., Quinn, B. M., Bard, A. J., "Electrochemistry of CdS nanoparticles: a correlation between optical and electrochemical band gaps," J. Am. Chem. Soc.123(36), 8860-8861 (2001).

[17]Liu, J., Yang, W., Li, Y., Fan, L., Li, Y., "Electrochemical studies of the effects of the size, ligand and composition on the band structures of CdSe, CdTe and their alloy nanocrystals," Phys. Chem. Chem. Phys. 16(10), 4778-4788 (2014).

[18] Kuçur, E., Bücking, W., Nann, T., "Electrochemical determination of mesoscopic phenomena, defect states in CdSe nanocrystals and charge carrier manipulability," Microchim. Acta.160(3), 299-308 (2007).

[19] Guyot-Sionnest, P., Wang, C., "Fast voltammetric and electrochromic response of semiconductor nanocrystal thin films," J. Phys. Chem. B 107(30), 7355-7359 (2003).

[20] Myung, N., Ding, Z., Bard, A. J., "Electrogenerated chemiluminescence of CdSe nanocrystals," Nano Lett. 2(11), 1315-1319 (2002).

[21]Boehme, S. C., Wang, H., Siebbeles, L. D. A., Vanmaekelbergh, D., Houtepen, A. J., "Electrochemical charging of CdSe quantum dot films: dependence on void size and counterion proximity," ACS Nano 7(3), 2500-2508 (2013).

[22] Shim, M., Guyot-Sionnest, P., "n-type colloidal semiconductor nanocrystals," Nature 407, 981-983 (2000).

[23] Spittel, D., Poppe, J., Meerbach, C., Ziegler, C., Hickey, S. G., Eychmüller, A., "Absolute energy level positions in CdSe nanostructures from potential-modulated absorption spectroscopy (EMAS)," ACS Nano 11(12), 12174-12184 (2017).

[24] Jha, P. P., Guyot-Sionnest, P., "Trion decay in colloidal quantum dots," ACS Nano 3(4), 1011-1015 (2009)

[25] Boehme, S. C., Walvis, T. A., Infante, I., Grozema, F. C., Vanmaekelbergh, D., Siebbeles, L. D. A., Houtepen, A. J., "Electrochemical control over photoinduced electron transfer and trapping in CdSe-CdTe quantum-dot solids," ACS Nano 8(7), 7067-7077 (2014).

[26]Žídek, K., Zheng, K., Chábera, P., Abdellah, M., Pullerits, T., "Quantum dot photodegradation due to CdSeZnO charge transfer: Transient absorption study,” Appl. Phys. Lett. 100(24), 243111 (2012).

[27] Puntambekar, A., Wang, Q., Miller, L., Smieszek, N., Chakrapani, V., "Electrochemical charging of CdSe quantum dots: Effects of adsorption versus intercalation," ACS Nano 10(12), 10988-10999 (2016).

[28]Zheng, K., Žídek, K., Abdellah, M., Chen, J., Chábera, P., Zhang, W., Al-Marri, M. J., Pullerits, T., "High excitation intensity opens a new trapping channel in organic-inorganic hybrid perovskite nanoparticles," ACS Energy Lett. 1(6), 1154-1161 (2016). 